\title{
Blocking Pseudomonas Aeruginosa, Chromobacterium Violaceum, and Ralstonia Solanacearum Adhesion by Fruit Glycans
}

Ofra Rachmaninov, Keren D. Zinger-Yosovich and Nechama Gilboa-Garber*

The Mina \& Everard Goodman Faculty of Life Sciences, Bar-Ilan University, Ramat-Gan 52900, Israel

Received: March 14, 2014; Accepted: April 23, 2014; Published: May 10, 2014

*Corresponding author: Nechama Gilboa-Garber, The Mina \& Everard Goodman Faculty of Life Sciences, Bar-Ilan University, Ramat-Gan 52900, Israel, Tel: 972-3-5317249; Fax: 972-3-5247346; E-mail: Nechama.Garber@biu.ac.il

\begin{abstract}
The soil-borne pathogens Pseudomonas aeruginosa Chromobacterium violaceum, and Ralstonia solanacearum, possess the lectins PA-IL, PA-IIL, CV-IIL, RSL, and RS-IIL, which may mediate their adhesion onto animal and plant target cells, enabling infections. Such infections may be prevented by surrounding the sensitive cells with competing glycans, which act as glycodecoys that block patholectins and capture pathogens that bear them. The abovementioned five lectins have been used by us as probes to reveal progeny-protecting glycodecoys in avian eggs, milk, royal jelly, and seeds. Herein we describe their usage as probes for fruit and onion glycodecoys. They revealed lectin-blocking galactosides, fructose, oligo/polysaccharides, and glycoproteins in most of the examined fruits. Galactose/arabinose- bearing compounds were detected by PA-IL in banana, carob, pineapple, pomegranate, kiwifruit, and dates. Diverse mannose/fucose-bearing compounds were detected by PAIIL in banana, onion, and pomegranate; by CV-IIL in pineapple; by RSL in banana, carob, date, onion, and pineapple, and by RS-IIL in date and fig. The results show the high efficiency of these lectins as probes for natural infection-preventing glycodecoys. Usage of fruit and seed embryo-protecting glycodecoys, unless allergenic, is advantageous for preventing animal intestinal and external and plant wilting infections since they are natural, harmless, inexpensive, and widely available.
\end{abstract}

Keywords: Anti-infection; Fruit glycans; Soil-borne pathogens; Pseudomonas aeruginosa; Ralstonia solanacearum

\section{Introduction}

The worldwide Pseudomonas aeruginosa and tropicalsubtropical Chromobacterium violaceum and Ralstonia solanacearum are soil saprophytic bacteria that aggressively decompose dead organisms and debris. Occasionally the first two are transformed into opportunistic animal (human) pathogens $[1,2]$. Clinical evidences describe these bacteria as a significant source of bacteria in burn victims, urinary-tract infections in catheterized patients, and hospital-acquired pneumonia in patients on respirators. Ralstonia solanacearum is well known devastating plant pathogen [3] wilting over 200 diverse species, causing heavy agricultural losses [4]. The emergence of these bacteria as a major opportunistic pathogens might be the outcome of their resistance to the antibiotics and disinfectants.

P. aeruginosa produces two lectins: PA-IL (LecA) (that bind galactose [Gal] and L-arabinose [Ara]), and PA-IIL (LecB) (that binds fucose [Fuc] > mannose [Man] and fructose [Fru]) $[5,6]$. These lectins contribute to the pathogens adhesion to target cells [7] and amplify the destructive effects of their accompanying virulence factors on those cells [8]. They also contribute to the pathogen auto-aggregation into biofilms [6], increasing its adhesion to victim host tissues and hampering antibacterial agent penetration into its cells [9].

C. violaceum possesses a fucophilic lectin, CV-IIL, homologous to PA-IIL in structure and displaying similar specificity (Fuc > Man and Fru) with preferential selectivity for Fuc $\alpha 1,2-$ epitope [10].

R. solanacearum produces two lectins: a Man>Fuc-binding lectin, RS-IIL [11], homologous to PA-IIL in structure, and a fucophilic lectin, RSL [12,13], of special propeller-like structure [14]. These lectins may mediate the pathogen adherence to seedling cell-wall saccharides and xylem, leading to wilting infections.

Infections by these pathogens might be prevented by cellsurrounding glycans that act as decoys blocking the patholectins. Anti-adhesion therapies for bacterial infections offer an alternative mechanism of action which prevents the host harm $[15,16]$. Such glycodecoys are especially abundant around embryos and are supplied to neonates [17]. They include monosaccharides (e.g. seminal fluid and honey fructose protecting spermatozoa and bee larvae); disaccharides (e.g. milk lactose), and oligosaccharides bearing terminal Gal, Fuc, Man or Ara (in milk, saliva, seminal and other body/plant fluids). The oligosaccharides may be free or linked to short peptides, polysaccharides (PSs) or glycoproteins (GPs). The described knowledge might extrapolate to a wide range of clinical applications: inhibition of lectins - host receptors interaction, vaccination, use of food ingredients which involved in host-lectin interactions, composition of nature ingredient 
and subminimal antibiotics concentrations and manipulation of hydrophobic glycodecoys [18-20].

Due to the crucial contribution of $P$. aeruginosa lectins PA-IL and PA-IIL to its pathogenicity, they have been widely used as authentic probes for searching natural [6] and synthetic [21-23] glycodecoys for blocking the pathogen binding to target cells. PA-IL and PA-IIL were highly useful for glycodecoy detection in human body fluids [6], avian egg whites [24,25], milks [26,27], royal jelly and honey [28]. Further on, CV-IIL, RSL, and RS-IIL were added to the scanning lectin battery -for glycodecoy search in those animal substances, floral nectars, and edible seeds [6,2931].

The current study aim was glycodecoy detection in edible fruit and onion extracts for the following purposes: 1) exhibition of the universality of "glycodecoy contribution to endogenous embryo protection against infections"; 2) application of the authentic patholectins as probes for discovering natural glycodecoys; 3) determination of natural glycodecoy protective capacity; and 4) choosing the best plant formulas for seedling protection against $R$. solanacearum and animal protection against $P$. aeruginosa gastrointestinal [32] and external infections. PA-IL, PA-IIL, CVIIL, RSL, and RS-IIL - blocking by non-dialyzed and dialyzed fruit extracts, differentiating between low ( $<10 \mathrm{kDa}, \mathrm{LMM})$ and high molecular mass(>10 kDa, HMM) glycans was examined using hemagglutination-inhibition (HAI) test. The extracted GPs were analyzed by Western blotting $(\mathrm{Wb})$ using the five bacterial lectins labeled by horseradish peroxidase.

\section{Results}

All the fruit and onion extracts inhibited (diversely) the five patholectin hemagglutinating activities (Figures 1 and 2, top panels). Inhibiting activities due to LMM $(<10 \mathrm{kDa})$ components were removed by dialysis, retaining the HMM activities.

As seen in Figure 1, PA-IL was blocked by most of the fruit LMM and HMM (PSs and GPs) components (top panels). It was most strongly blocked by banana, carob, pineapple, and pomegranate, followed by kiwifruit, while least sensitive to blocking by onion extract. It also stained their GPs (Wbs in the respective bottom panels), most strongly and widely the date GP bands (around 10 heavy bands at 25-105 kDa), followed by pineapple (1 heavy band at around $25 \mathrm{kDa}$ and a compact bandcluster at 150-250 kDa), carob and onion (about five GP bands each at 40-105 kDa), fig (a sole band at $25 \mathrm{kDa}$ ), kiwi (2 bands at 20-30 kDa), and terminating in pomegranate (1 pale GP band). It did not stain any banana or grape GP.

Figure 1 shows that PA-IIL, was more sensitive than PAIL to all the extracts examined. It was most strongly blocked by pomegranate, grape, and kiwifruit, followed by date, carob, banana, and pineapple. It also nicely reacted with onion and fig. Like PA-IL, PA-IIL strongly stained a wide spectrum of the fruit extract GPs, excluding grapes. Unlike PA-IL, it did react with banana and pomegranate GPs, displaying considerably more pronounced staining of onion and pomegranate GP bands.

CV-IIL sensitivity to the fruits was considerably weaker, and unlike the other lectins, it was not inhibited at all by onion (Figure 1 , top panel). It was most sensitive to inhibition by kiwifruit and pineapple, followed by pomegranate, grape, carob, and date. CV-IIL stained only few fruit GPs (Figure 1, bottom panel), most strongly those of pineapple.

As seen in Figure 2, the R. solanacearum lectin RSL, was quite sensitive to blocking by the fruit glycans (top panel) being most strongly blocked by extracts of pomegranate, followed by date, kiwifruit, grape, pineapple, and carob. This lectin strongly stained many GPs (ranging from 15 to $250 \mathrm{kDa}$ ) in the date, onion, carob, banana, and pineapple $\mathrm{Wbs}$ (much more than the other lectins), but no grape and negligible fig GP bands were stained by it.

Figure 2 further shows that RS-IIL was the most sensitive lectin to the fruit extracts, being most strongly inhibited by all of them and unique for its strongest interactions with the fig

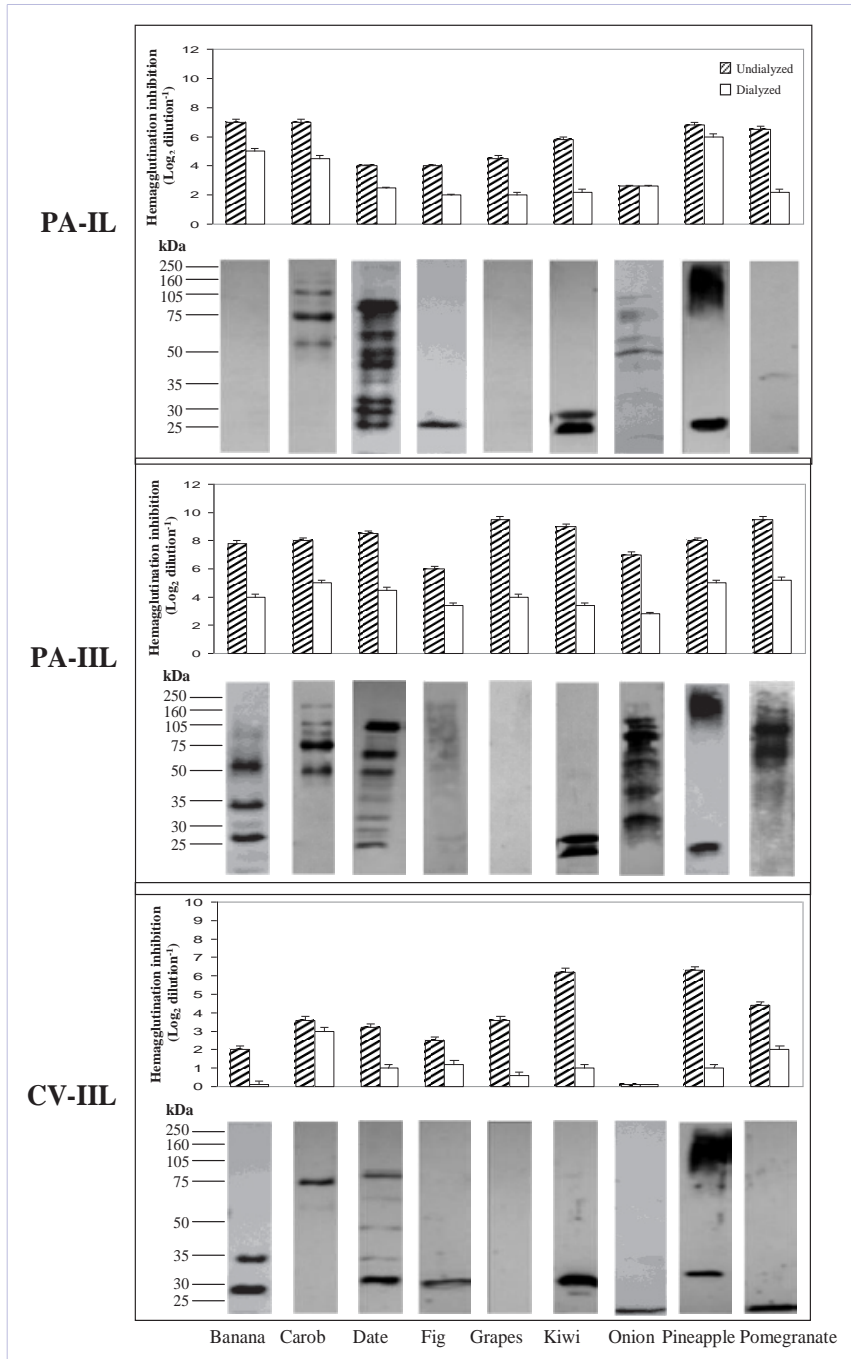

Figure 1: Interactions of fruit and onion extracts with P. aeruginosa galactophilic and fucophilic lectins PA-IL and PA-IIL (respectively) and C. violaceum fucophilic lectin CV-IIL. The interactions were examined by inhibition of the lectin hemagglutinating activities by nondialyzed and dialyzed samples of the extracts (top panels) and by Western blotting $(\mathrm{Wb})$ using the same lectins labeled by peroxidase (bottom panels). 

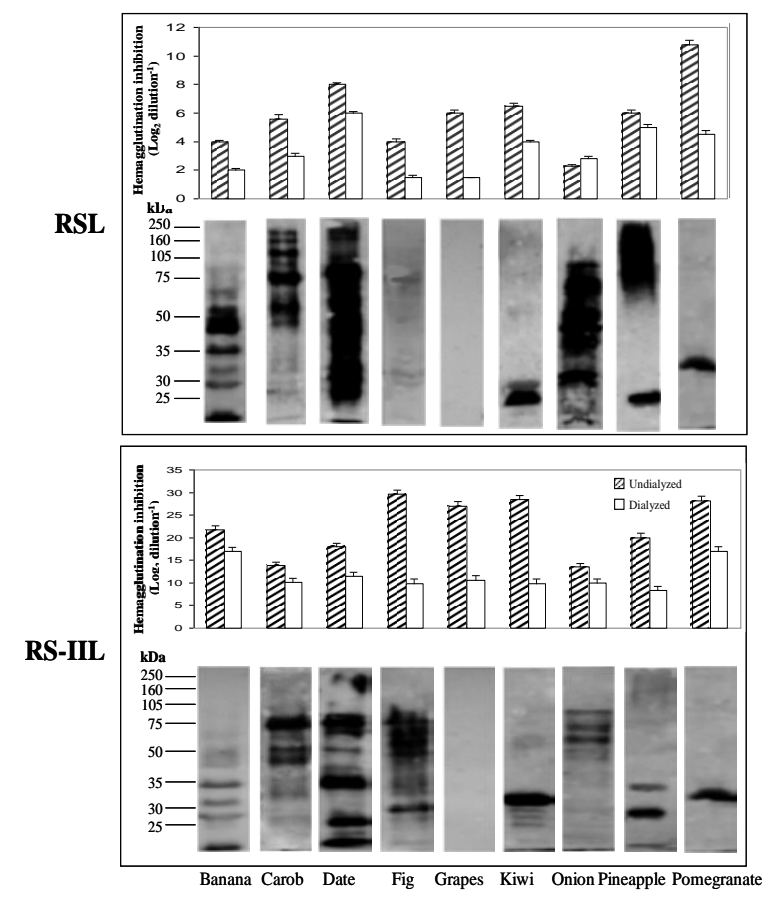

Figure 2: Interactions of fruit and onion extracts with R. solanacearum mannophilic (>fucophilic) RS-IIL and fucophilic (>mannophilic) RSL lectins. The interactions were examined by inhibition of the lectin hemagglutinating activities by nondialyzed and dialyzed samples of the extracts (top panels) and by Western blotting ( $\mathrm{Wb}$ ) using the same lectins labeled by peroxidase (bottom panels).

extract glycans (which reacted most weakly with the other lectins), including special staining of the fig 25-80 kDa GPs. RSIIL was also most strongly blocked by kiwifruit, pomegranate, and grape, followed by banana, pineapple, and date, and it also showed a clear sensitivity to carob and onion. This lectin stained many date, carob, and banana GP bands, in addition to the unique rich staining of the fig GPs. Like the other lectins, it also did not stain grape GPs, while staining few kiwi GPs. Unlike PA-IIL and RSL, RS-IIL stained only a few onion GPs and was profoundly outstanding in not staining the pineapple HMM GP cluster (75$250 \mathrm{kDa}$ ), which was clearly stained by the other four lectins.

\section{Discussion}

Prevention of pathogenic bacterial infections in animals and plants is a major goal in medicine, veterinary medicine, and agriculture. Since most pathogenic bacteria have become antibiotic-resistant, special efforts are invested in preventing infections by hampering their adhesion to the target cells.

Natural anti-infective tactics hamper lectin-dependent pathogen binding to cells by surrounding the sensitive cells with glycodecoys that compete with the cell receptor saccharides [32-34]. These glycodecoys not only block lectin binding to the sensitive target cells, but also capture the lectin-bearing bacteria, preventing their adhesion to the target cells via additional adhesins. This strategy protects immuno-immature animal and plant embryos, neonates, and sensitive adult cells (including germ cells) against infections.

The patholectins PA-IL and PA-IIL of P. aeruginosa, CV-IIL of C. violaceum, and RSL and RS-IIL of $R$. solanacearum, were shown by us during the last decade to be highly useful authentic probes for detection of glycodecoys in avian egg whites [6,24,25], milk [27] royal jelly and honey [6,28] and seed glycans [29-31] (Table $1)$.

The present report describes their usage for the study of plant-glycodecoy presence in extracts of eight fruits and onion. The fruits were chosen because they harbor seeds, they contain non-starch PSs that bear glycodecoy-type glycans (their LMM and multivalent polymeric glycans, including PSs and GPs, fit that function), they are not expensive, and are widely consumed food stuffs, generally without adverse effects, except allergies [35-37].

Each fruit extract was examined without fractionation for determination of total lectin- blocking activities of both LMM and HMM glycans, and following removal of LMM $(<10 \mathrm{kDa})$ by dialysis - for evaluation of the contribution of the retained HMM fraction to lectin blocking.

While leguminous seed glycans generally inhibited PA-IL more strongly than the Fuc/Man-binding patholectins [29] the fruit and vegetable extracts inhibited the other four lectins more than PA-IL.

The concentration of potential PA-IL-blocking monomeric Gal and Ara in fruits is very low: grapes contain 7.5-16, kiwi 27, and onion $15 \mathrm{mg} \mathrm{Gal} / 100 \mathrm{~g}$ fresh weight. However, the Galcontaining disaccharide melibiose (Gal $\alpha 1,6 \mathrm{Glc}$ ), trisaccharide raffinose (Gal $\alpha 1,6 \mathrm{Glc} \beta 1,2 \mathrm{Fru}$ ), and tetrasaccharde stachyose (Gal $\alpha 1,6 \mathrm{Gal} \alpha 1,6 \mathrm{Glc} \beta 1,2 \mathrm{Fru}$ ) are abundant. Raffinose is one of the most abundant trisaccharides in nature, widely occurring in plants (like sucrose), and stachyose coexists with them [38]. These saccharides are strong inhibitors of PA-IL: melibiose, raffinose, and stachyose are x13.3, x4.4 and x5.7, respectively, more potent inhibitors of PA-IL than monomeric Gal [39]. The Gal/Ara-bearing PSs: galactomannans, or galactoglucomannans (Table 2 [2]) and arabinogalactans, which are potent PA-IL inhibitors, are major fruit primary cell-wall PSs existing in plants in high "concentrations": "xyloglucans", which are comparatively homogeneous PSs made of glucan backbone substituted with xylosyl side chains, also occasionally bear galactose residues (Table 2 [5]). GPs that coexist with them at low concentrations only weakly contribute to PA-IL blocking in plants, contrary to their major function in animal embryo- and neonate- protection $[6,24]$.The Wb data (Figure 1, bottom panels) show that PA-ILinhibiting activities of banana, grape, and pomegranate HMM glycans are not dependent on their GPs since no band-staining with PA-IL was observed in their Wbs. The GPs of the other fruits attracted PA-IL, which moderately stained a few carob and onion GP bands, while very strongly and widely staining around 10 heavy date GP bands, but only few fig and kiwifruit bands.

PA-IIL and CV-IIL inhibitors include free fructose, mannosylated and fucosylated PSs, and GPs possessing Man and Le (a) or $\mathrm{H}$ epitope-bearing antennae (Table 2). The last two 
Table 1: Inhibition of the hemagglutinating activities of the five patholectins by avian egg white, milk, royal jelly, and seed glycodecoys - all of them protecting sensitive animal offsprings (presented as HAI $\log _{2}$ dilution ${ }^{-1}$ ).

\begin{tabular}{|c|c|c|c|c|c|}
\hline Embryo/neonate- protecting medium: Lectin & PA-IL & PA-IIL & CV-IIL & RSL & RS-IIL \\
\hline Chicken egg white & 0 & 4 & 3 & 1 & 4 \\
\hline Quail egg white & 0 & 15 & 23 & 6 & 22 \\
\hline Pigeon egg white & 22 & 16 & 15 & 4 & 19 \\
\hline Human milk & 8 & 19 & 8 & 15 & 10 \\
\hline Cow milk & 7 & 1 & 2 & 3 & 4 \\
\hline Royal jelly & 6 & 14 & 11 & 6 & 13 \\
\hline Seeds* (cashew, cocoa, coffee, pumpkin) & 7.5 & 6 & 1.2 & 4 & 11.5 \\
\hline
\end{tabular}

*The values are means of the four seeds. Highest HAI values are in bold.

epitopes are strong ligands of PA-IIL and CV-IIL, respectively.

Fructose, which blocks PA-IIL, CV-IIL, RSL, and RS-IIL, exists in fruits as a monomer and as the most abundant disaccharide sucrose. Mannose is rare as a monomer; there are limited amounts of fruit mannans, and oligomannosylated N-glycans are present only in minor amounts [40,41]. The contribution of the plant GPs to this lectin-blocking is low as opposed to the mannosylated animal GPs (e.g., quail egg albumen and royal jelly) $[25,28]$, which strongly block PA-IIL.

Fuc, which also blocks the same four lectins, is present in plant PSs as Fuc $\alpha 1$,2- [42] linked to Gals of xyloglucans, which occasionally bear galactose or fucosyl-galactose residues (Table 2 [5]). It exists in GPs as core Fuc $\alpha 1,3$ - bound to Man(2) Xyl-bearing $\mathrm{N}$-glycans (MUX, Table $2[7,8]$ ), which strongly attract RSL, or as a single Fuc $\alpha 1,4-$ [Le](a) epitope that strongly attracts PA-IIL (Table 2 [13]). PA-IIL and RSL may also bind to complex N-glycans bearing both Le(a) and core Fuc $\alpha 1,3 / 6$ - branches (Table 2 [12]). Among the mannosylated/fucosylated GP N-glycans, the most abundant ones bear a 31,2 -xylosyl and a core alpha 1,3Fuc residue linked to the terminal $\mathrm{N}$-acetylglucosamine, similar to those of the major $\mathrm{N}$-linked oligosaccharides of horseradish peroxidase and pineapple stem bromelain (Table 2 [7,8], MUXF and MMXF).

The sensitivity of $R$. solanacearum lectin RS-IIL to the fruit glycans was highest (Figure 1,2), like the outstanding sensitivity of PA-IIL to human milk $[43,44]$. Including the mannophilic plant lectin Con A with the bacterial lectins (Table 3) shows that PA-IIL and RS-IIL share with it insensitivity to the plant galactomannans vs. high sensitivity to yeast mannan. RS-IIL is closer to Con A than to PA-IIL in its lower sensitivity to human milk GPs and its insensitivity to the milk Le (a) epitope.

Taking the kiwifruit (Actinidia deliciosa) as an example, Schroder et al. [45] reported that the galactoglucomannan from the primary cell walls of the ripe fruit is mainly composed of alternating Glc $\beta 1,4$ Man backbone, with approximately one third of it carrying either single Gal $\alpha 1,6$ or Gal $\beta 1,2$ Gal $\alpha 1$-. Its neutral arabinogalactan $(7 \%)$ was proposed to contain a highly branched galactan with alternating 1,3- and 1,6-Gal backbone and 1,3- and 1,6-Gal side chains terminating with 1,2-Ara residues. Its major
(93\%) acidic arabino rhamnogalacturonan was shown to be mainly composed of rhamnogalacturonan backbone-bearing terminal 1- $\alpha-\mathrm{L}$ Ara and Gal residues as side chains $[46,47]$. These fruit GPs also bear oligosaccharide antennae with terminal Gal, Man, and Fuc (Table 2), including the Le (a) epitope $[35,48,49]$, who diligently examined plant-glycan involvement in food allergies and cross-allergic reactions between diverse plant products, described data of 27 plant foodstuffs, showing that the glycans of these foodstuffs ranged from the typical vacuolar "horseradish peroxidase" type and oligomannosylated types (Table 2 [8 and 9]) to complex Le(a)-carrying structures (Table 2 $[11,12])$. Apple, banana, carrot, kiwi, and onion, as well as other fruits (out of the scope of our research), were found by them to be particularly rich in Le (a)-carrying N-glycans. They concluded that the Le (a)-type structure is very widespread among plants, excluding cauliflower. The presence of Le (a) in fruits resembles its presence in human milk. Its outstandingly high affinity to PAIIL $[43,44]$ is in agreement with the view that it might contribute to the protection of the fruit embryos against PA-IIL-bearing $P$. aeruginosa, as does human milk. Comparison of the fruit and onion patholectin-inhibiting efficiencies towards the five lectins (Figures 1 and 2, top panels and Table 3) has shown that pomegranate and kiwi, followed by pineapple, grapes, and dates, possess the overall strongest patholectin-blocking activities. Banana and carob, followed by pineapple, pomegranate, and kiwifruit, were most efficient for blocking PA-IL; pomegranate and grapes, followed by kiwi and dates, were most efficient for binding PA-IIL; kiwifruit and pineapple, followed by pomegranate, were most effective CV-IIL inhibitors; and pomegranate, followed by dates, kiwi, and grapes, were the strongest RSL inhibitors. RSIIL was unique in being most sensitive to fig glycans, followed by those of kiwi, pomegranate, grapes, banana, and pineapple. Comparison of the fruit interactions with the diverse lectins showed a very interesting analogy between banana and carob extracts in regard to the two P. aeruginosa lectins and Con A (Table 3). Both most strongly blocked PA-IL. Their identical inhibitions of PA-IIL (Table 3) were probably due to their fructose, Man-bearing compounds, and Fuc-bearing Le (a) [35]. Wilson et al. documented the presence of Le (a) in banana. The banana and carob interactions with the other four lectins diverged: banana exerted a stronger inhibition of the mannophilic 
Table 2. Proposed structures of natural PSs and N-glycans that block the five patholectins.

\begin{tabular}{|c|c|}
\hline Name (abbreviation) & Proposed Structure \\
\hline $\begin{array}{l}\text { 1) Galactosylated pectin } \\
\text { (GalA = galacturonic acid) } \\
\text { (Rha = rhamnose) }\end{array}$ & $\begin{array}{c}-\mathrm{D}-\mathrm{Gal} A \alpha 1,2 \mathrm{~L}-\mathrm{Rha} \alpha 1,4 \mathrm{D}-\mathrm{Gal} A- \\
\uparrow \mathbf{1 , 4} \\
\mathbf{G a l \beta} \\
\pm \text { L-Araa1,3/6 } \\
\end{array}$ \\
\hline 2) Galacto/galactogluco-mannan & 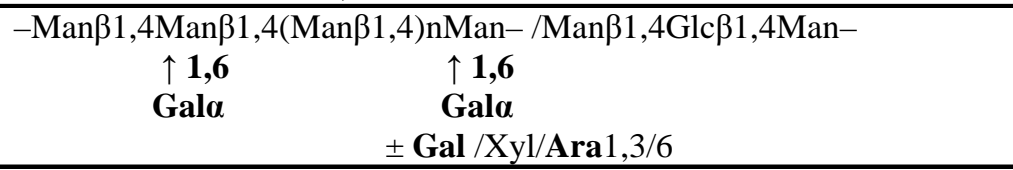 \\
\hline $\begin{array}{l}\text { 3) A rabinogalactan } \\
\text { (GIcA = Glucuronic acid) }\end{array}$ & 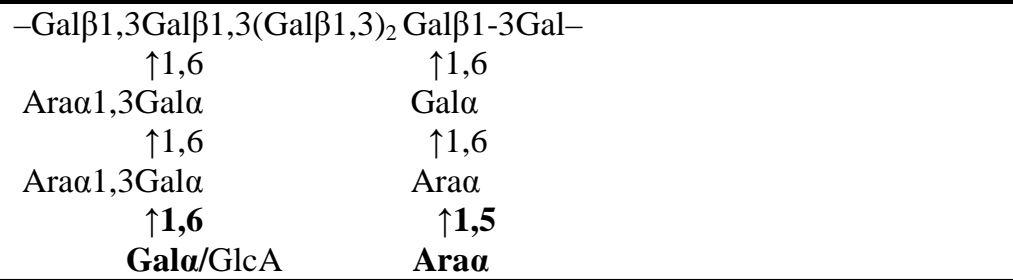 \\
\hline 4) Mannan & 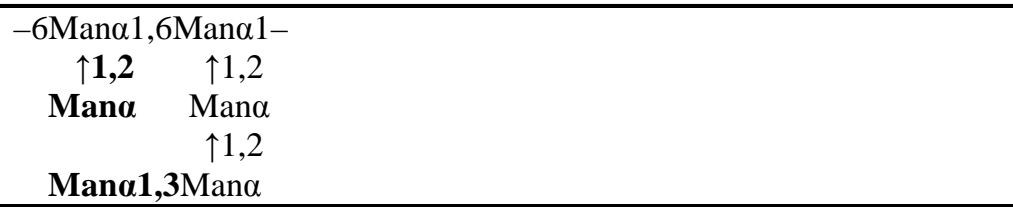 \\
\hline 5) Fucosylated xyloglucans & 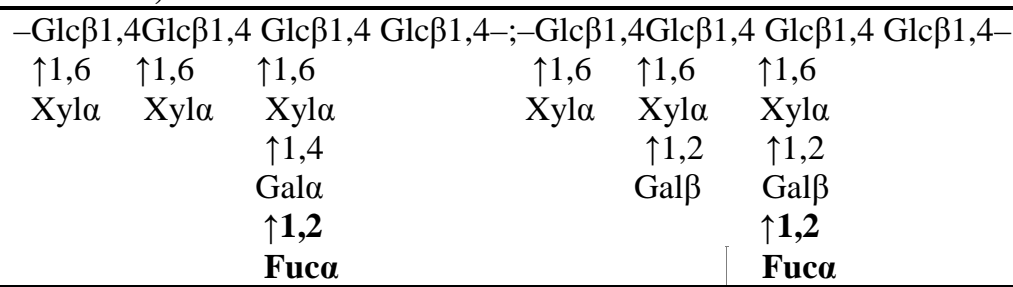 \\
\hline 6) Oligo (3) mannosylated N-glycan & 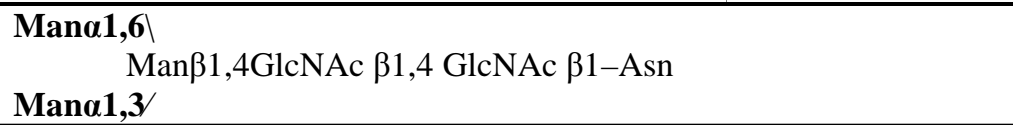 \\
\hline $\begin{array}{l}\text { 7) Bromelain-type N-glycan } \\
\text { MUXF }^{3}\end{array}$ & 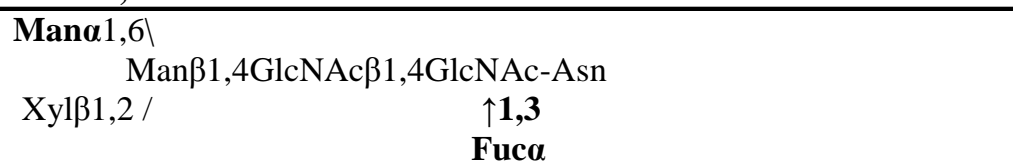 \\
\hline $\begin{array}{l}\text { 8) Horseradish peroxidase-type } \\
\mathrm{N} \text {-glycan } \mathrm{MMXF}^{3}\end{array}$ & 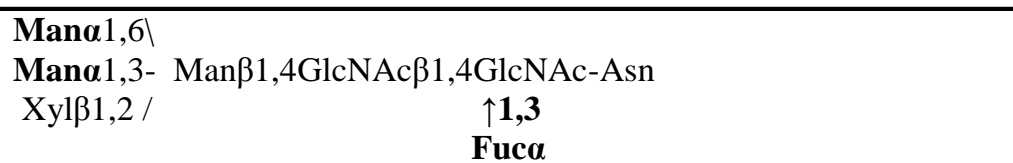 \\
\hline $\begin{array}{l}\text { 9) Highly (9) mannosylated } \\
\mathrm{N} \text {-glycans }\end{array}$ & 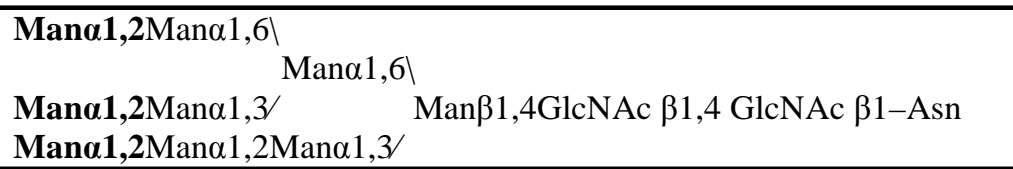 \\
\hline 10) $\mathrm{H}$ blood group trisaccharide & Fuca1,2G al $\beta 1,3 / 4 \mathrm{GICNAC-}$ \\
\hline 11) Lewis(a) tetrasaccharide & $\begin{array}{c}-\mathrm{Gal} \beta 1,3 \mathrm{G} \mid \mathrm{cN} A \mathrm{~A} 1,3 \mathrm{G} \text { al - } \\
\uparrow \mathbf{1 , 4} \\
\mathbf{F u c \alpha} \\
\end{array}$ \\
\hline $\begin{array}{l}\text { 12) Lewis(a) and core } \\
\text { Fuc } \alpha 1,3 / 6\end{array}$ & 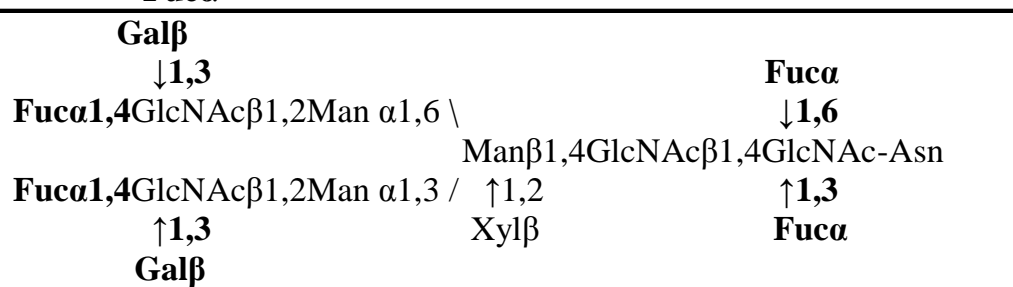 \\
\hline
\end{tabular}


RS-IIL while carob was a stronger inhibitor of the fucophilic CVIIL and RSL. The banana and carob Wb data (presented in Figures 1,2 , bottom panels) seem to indicate that the blocking terminal Gal $1-6$ residues in banana are merely associated with oligosaccharides and PSs rather than with GPs, since no PA-ILstained GP bands were observed in the banana extract $\mathrm{Wb}$. This was in contrast to the clear PA-IL staining of the carob and several other fruit GPs, and as opposed to the staining of banana GP bands by the other four lectins (Figures 1,2, bottom panels). Hence, it may be deduced that the contribution of banana, as well as grape and pomegranate GPs, to PA-IL blocking, is negligible (Figure 1, bottom panel). However, in contrast to the total absence of GP bands in grapes, banana GPs are stained by the other four lectins. Two banana GP bands (at around 28 and 34 $\mathrm{kDa}$ ), which were selectively stained by CV-IIL, and also strongly by PA-IIL and RSL, might bear H-type Fuc $\alpha 1$,2 epitope (which is an important ligand of these three Fuc- specific lectins). A third GP band (at around $50-55 \mathrm{kDa}$ ) strongly stained by PA-IIL and RSL, can bear the Le (a) epitope, which had already been documented $[35,36]$ and core Fuc, respectively. The banana Wbs showed additional GPs (at a range of around 40 to $50 \mathrm{kDa}$ ) that were strongly and selectively stained by RSL (probably due to core Fuc3) and additional GPs that were more weakly stained by PA-IIL, RSL, and RS-IIL. Despite stronger blocking of RSL by carob than by banana, their Wbs with RSL seemed to be equal (at a range of 30 to $250 \mathrm{kDa}$ ), differing in the molecular mass distribution of the more conspicuous GP bands. The extracted date and fig glycans shared relatively high affinity to the mannophilic RS-IIL and low PA-IL binding, but differed in their interactions with the other three fucophilic lectins and in their Wbs. As may be seen in Figures 1 and 2 (top panels) and Table 3, the fig-extract blocking of PA-IIL, CV-IIL, and ConA were weaker than those of the date. The Wbs of these 2 fruit extracts were also very interesting (Figures 1,2, bottom panels). The date GP glycans, as compared to the other eight fruits, displayed strongest, richest and widest GP band reactivity with all five patholectins indicating the presence of both terminal Gal (PA-IL-stained) and Fuc/Man (stained by the four lectins) residues. The fig extract blocking of the five lectins is probably due to their fructose, galactosylated and mannosylated oligosaccharides, and PSs, which selectively most strongly blocked the mannophilic RS-IIL.
However, in contrast to dates, its Wbs were relatively poor: only the RS-IIL-stained Wb showed considerable GP band staining, while only one GP band was strongly stained by PA-IL and CV-IIL. The high sensitivity of RS-IIL to fig N-glycans seems to indicate the presence of an important RS-IIL ligand on them. This is a significant preliminary finding deserving further study. The grape-extract strongest blocking of PA-IIL, RS-IIL, and Con A, followed by RSL, is very similar to the date pattern (Table 3). This may be due to fructose and mannosylated PSs but not GPs since, in contrast to the rich date GP staining, no GP bands were observed in any of the grape Wbs (Figures $1 \& 2$ bottom panels). The grape-negative $\mathrm{Wbs}$, with all the lectins, were outstanding among the herein-examined fruits but similar to our previous results with cocoa-seed extracts [30,31]. Kiwifruit and pineapple represent another pair of fruits being similar in their very strong interactions with almost all the examined lectins and with special preference for CV-IIL (Figures 1 \& 2 Table 3). The pineapple inhibition of PA-IL is stronger, and of PA-IIL -weaker than those of the kiwifruit, which was reported to contain Le (a) epitope [35]. Strongest inhibition of CV-IIL (Table 3) could be attributed to fructose, oligosaccharides, and H-type Fuca1,2-bearing xyloglucans since their Wbs presented relatively poor lectinstained GP band repertoire. Two GP bands in the kiwi and one band in the pineapple (at a range of 20 to $30 \mathrm{kDa}$ ) were stained by the five patholectins (Figs. 1,2 bottom panels). In addition, in the pineapple, there was a cluster of bands at 75-250 kDa equally stained by CV-IIL, PA-IL, and PA-IIL, with stronger staining by RSL but almost no staining with RS-IIL. The strong PA-IL blocking activity of kiwi and pineapple might be mainly attributed to their above-described Gal/Ara-containing PSs [46,47]. Figures 1,2 show another very interesting observation - strongest overall lectin- blocking activities of the pomegranate without considerable GP contribution - versus the weakest overall lectinblocking of the onion (not inhibiting CV-IIL and only weakly inhibiting PA-IL and RSL) apart from strong blocking of PA-IIL, followed by RS-IIL, associated with rich staining of its GP bands by both PA-IIL and RSL (at a range of 20 to105 kDa). The combined PA-IIL and RSL binding to the onion GPs is in accord with the description of N-glycans that bear both core Fuc and Le (a) (Table 2[12]), and in accord with the Wilson et al. report on Le (a) epitope presence in onion N-glycans. Since its GP bands were

Table 3: Comparison of the relative inhibitory potential of each of the eight fruit and onion extracts for the five patholectins (with ConA as a reference mannophilic lectin\}.

\begin{tabular}{|c|c|c|c|c|c|c|c|}
\hline & PA-IL & PA-IIL & CV-IIL & RSL & RS-IIL & Sum & ConA \\
\hline Banana & 100 & 80 & 30 & 35 & 75 & 320 & 55 \\
\hline Carob & 100 & 80 & 55 & 05 & 42 & 327 & 55 \\
\hline Date & 55 & 90 & 50 & 80 & 58 & 333 & 98 \\
\hline Fig & 55 & 60 & 40 & 35 & 100 & 290 & 45 \\
\hline Grapes & 60 & 100 & 52 & 54 & 90 & 358 & 76 \\
\hline Kiwi & 78 & 96 & 100 & 56 & 95 & 425 & 47 \\
\hline Onion & 32 & 70 & 0 & 23 & 42 & 167 & 43 \\
\hline Pineapple & 95 & 85 & 100 & 53 & 60 & 393 & 45 \\
\hline Pomegranate & 91 & 100 & 70 & 100 & 95 & 456 & 100 \\
\hline
\end{tabular}


also weakly (identically) stained by PA-IL and RS-IIL, it seems that they also possess Gal- and Man/Fuc-bearing N-glycans. Taken together, the data of the herein presented research of fruit and onion glycodecoys by means of five authentic patholectins (two of $P$. aeruginosa, one of $C$. violaceum, and two of $R$. solanacearum) add important information related to plant embryo-protection against infections, which is also relevant to humans, other animals, and plants. Owing to the selective glycan specificities of the five patholectins, they have been proven to be powerful probes for the identification of diverse anti-infective glycodecoys in diverse organisms. Comparing the lectin binding by the plant and animal glycodecoys has enriched the perspective of this subject. Blocking PA-IL, PA-IIL, RSL, and RS-IIL by common epitopes of human milk, avian egg whites, beehive products, and fruits/onion most strongly indicates the importance of their consumption as food and their medical, veterinary and agricultural usage. Although almost all the examined fruit extracts inhibited the five lectins, with pomegranate displaying the highest patholectin-blocking activity, followed by kiwifruit, pineapple, grapes, and fig, PA-IL was most strongly blocked by banana and carob and RS-IIL was outstanding in its highest affinity to the fig, followed by kiwi, pomegranate, and grapes. The present results seem to indicate that in fruits as in the animalprotecting systems, both LMM and HMM glycans are important for blocking patholectins. However, in animals, the GP contribution is higher while in plants, the PSs are important. In addition to their efficiency as glycodecoy probes, the five patholectins might also be useful probes for detecting allergenic glycans. The plant embryo-protecting glycodecoys, unless allergenic, are advantageous for preventing animal (intestinal and external) and plant infections since they are natural, widely available, and consumed as mostly harmless, inexpensive edible plant products.

\section{Experimental Procedures}

\section{Lectin preparations}

The bacterial lectins: PA-IL, PA-IIL, CV-IIL, RSL, and RS-IIL, were isolated from cell extracts of P.aeruginosa ATCC 33347, C. violaceum (Bergonzini) ATCC 12472, and $R$. solanacearum ATCC 11696, purchased from the American Type Culture Collection (ATCC) (Manassas, Virginia), and purified, as described earlier [5,10-12]. Quality controls of the purified lectins were performed by SDS-PAGE with Coomassie brilliant blue staining.

\section{The fruit and onion extract preparations}

Extracts of fresh banana (Musa acuminata), carob (Ceratonia siliqua), date (Phoenix dactylifera), fig (Ficus carica), grape (Citrus paradisi), kiwi (Actinidia chinensis), pineapple (Ananas comosus), pomegranate (Punica granatum) and onion (Allium сера) were prepared by their chopping, suspension at $10 \%(\mathrm{~V} / \mathrm{V})$ concentration in $0.025 \mathrm{M}$ phosphate buffered (at pH 7.2) isotonic saline $(0.85 \% \mathrm{NaCl})$ solution (PBS), and homogenization in a food processor. Each suspension was centrifuged $(10,000 \mathrm{~g})$ for $10 \mathrm{~min}$ and the supernatant fluid was carefully collected. A half-volume of each supernatant was dialyzed overnight (using a dialysis membrane cut-off of $10 \mathrm{kDa}$ ) against x 500 volume of PBS at pH
7.2 (refreshed three times) at $5^{\circ} \mathrm{C}$. Both the non-dialyzed and dialyzed preparations were divided into several 1-mL aliquots for storage at $5^{\circ} \mathrm{C}$ for a few days or at $-20^{\circ} \mathrm{C}$ for longer periods.

Hemagglutinating activity (HA) determination: The lectin HA was determined using papain-treated human type $\mathrm{O}(\mathrm{H})$ red blood cells (erythrocytes). The erythrocytes (kindly obtained from the Magen David Adom National Blood Services in Israel) were washed 3 times with PBS, and then treated with $0.1 \%$ papain with $0.01 \%$ cysteine, as previously described [5]. A 50- $\mu \mathrm{L}$ sample of each bacterial lectin preparation examined (starting in $20 \mu \mathrm{gmL}-1$ solution) was serially 2 -fold diluted in tubes with 50 $\mu \mathrm{L}$ of saline. Then, saline and $5 \%(\mathrm{~V} / \mathrm{V})$ suspension of the papaintreated erythrocytes in saline (50 $\mu \mathrm{L}$ each) were added to each tube and thoroughly mixed. After $30 \mathrm{~min}$ at room temperature, the tubes were centrifuged for $30 \mathrm{sec}(1000 \times \mathrm{g})$, and HA was examined, as previously described [5]. The highest dilution leading to agglutination of all the erythrocytes in one mass was determined. The activity was represented by the number of tubes (2-fold dilutions) in which there was hemagglutination $\left(\log _{2}\right.$ dilution $^{-1}=7$ means 7 positive tubes $=$ positive reaction up to dilution of $1: 128$, or original activity of 128 hemagglutination (units).

Hemagglutination inhibition (HAI) test: In the HAI test, each seed extract was serially 2 -fold diluted in $50 \mathrm{~mL}$ of saline. Then $50 \mathrm{~mL}$ of the lectin solution (at highest dilution that agglutinates all the erythrocytes in one large mass) was added to each tube. After $30 \mathrm{~min}$ at room temperature, $50 \mathrm{~mL}$ of the papain-treated human 0 type erythrocyte suspension was added to each tube and HA was examined, as described above [5]. HAI intensity was represented by the number of 2 - fold dilutions ( $\log _{2}$ dilution $^{-1}$ ) without considerable hemagglutination preceding its reappearance.

Western blot (Wb) analyses: $\mathrm{Wb}$ analyses demonstrating the differential peroxidase-labled lectin interactions with individual SDS-PAGE-separated GPs, were performed as follows: $15-\mu \mathrm{L}$ samples of each fruit/onion extract at a concentration of around 1 mg.mL-1 were mixed 1:1 with a sample buffer, boiled, and applied to wells in 10\% SDS-PAGE (at $140 \mathrm{~V}$ ) in a MiniPROTEAN Cell 3 Electrophoresis (Bio-Rad, Hercules, California), as previously described [43]. Following SDS-PAGE, the proteins were transferred to a nitrocellulose $(0.45 \mu \mathrm{m}$, Bio-Rad $)$ membrane at $4{ }^{\circ} \mathrm{C}$ for $2 \mathrm{~h}(85 \mathrm{~mA} / 40-50 \mathrm{~V})$ using the Mini Trans-Blot Module (Bio-Rad). The membranes were incubated overnight in blocking buffer $0.02 \mathrm{M}$, PBS pH 7.2, containing 3\% bovine serum albumin and $0.05 \%$ Tween 20 , exposed to horseradish-peroxidaselabeled lectins (about $1 \mu \mathrm{g} / \mathrm{mL}^{-1}$, dissolved in the same blocking buffer with $0.1 \%$ Tween 20 ) at room temperature for $2 \mathrm{~h}$, and then thoroughly washed. The peroxidase reaction was visualized using enhanced chemiluminescence (Amersham International PLC, Buckinghamshire, UK) and recorded onto photographic films. Controls with the peroxidase-labeled lectins in the presence of a $0.3 \mathrm{M}$ concentration of the relevant blocking sugars in their reaction mixtures were used in parallel in order to rule out nonspecific (sugar-independent) lectin binding [43]. 
Statistical analysis: The HAI results were analyzed by Student's t-test. The data presented in the figures generally represent means \pm SE (standard errors of the means) of at least five experimental results for each lectin.

\section{Acknowledgements}

The authors express their gratitude to Ms Sharon Victor for her great help with editing and preparation of this manuscript and to Ms Ela Gindy for her great help in the graphic presentation. This work is part of the PhD thesis of Ofra Rachmaninov, Bar-Ilan University.

\section{References}

1. Shao PL, Hsueh PR, Chang YC, Lu CY, Lee PY, et al. (2002) Chromobacterium violaceum infection in children: A case of fatal septicemia with nasopharyngeal abscess and literature review. Pediatr Infect Dis J 21(7): 707-709.

2. Sadikot RT, Blackwell TS, Christman JW, Prince AS (2005) Pathogenhost interactions in Pseudomonas aeruginosa pneumonia. Am J Respir Crit Care Med 171(11): 1209-1223.

3. Saile E, McGarvey JA, Schell MA, Denny TP (1997) Role of extracellular polysaccharide and endoglucanase in root invasion and colonization of tomato plants by Ralstonia solanacearum. Phytopathology 87(12): 1264-1271.

4. Denny TP (2000) Ralstonia solanacearum--a plant pathogen in touch with its host. Trends Microbiol 8(11): 486-489.

5. Gilboa-Garber N (1982) Pseudomonas aeruginosa lectins. Methods Enzymol 83: 378-385.

6. Gilboa-Garber N, Zinger-Yosovich KD, Sudakevitz D, Lerrer B, Imberty A, et al. (2011) The five bacterial lectins: PA-IL, PA-IIL, RSL, RS-IIL, and CV-IIL- interactions with diverse animal cells and glycoproteins. Adv Exp Med Biol 705: 155-211.

7. Gilboa-Garber N (1994) Pseudomonas aeruginosa PA-I and PA-II lectins. In: Beuth J \& Pulverer G (Eds.), Lectin Blocking: New Strategies for the Prevention and Therapy of Tumor Metastasis and Infectious Diseases. Gustav Fischer Verlag, New York, pp. 44-58.

8. Gilboa-Garber N, Garber N (1989) Microbial lectin cofunction with lytic activities as a model for a general basic lectin role. FEMS Microbiol Rev 5(3): 211-221.

9. Gilboa-Garber N, Avichezer D, Garber NC (1997) Bacterial lectins: properties, structure, effects, function and applications. In: Gabius HJ \& Gabius S (Eds), Glycosciences: Status and Perspectives. Chapman and Hall, Weinheim, Germany, pp. 369-398.

10. Zinger-Yosovich K, Sudakevitz D, Imberty A, Garber NC, Gilboa-Garber N (2006) Production and properties of the native Chromobacterium violaceum fucose-binding lectin (CV-IIL) compared to homologous lectins of Pseudomonas aeruginosa (PA-IIL) and Ralstonia solanacearum (RS-IIL). Microbiology 152(Pt 2): 457-463.

11. Sudakevitz D, Kostlánová N, Blatman-Jan G, Mitchell EP, Lerrer B, et al. (2004) A new Ralstonia solanacearum high-affinity mannose-binding lectin RS-IIL structurally resembling the Pseudomonas aeruginosa fucose-specific lectin PA-IIL. Mol Microbiol 52(3): 691-700.

12.Sudakevitz D, Imberty A, Gilboa-Garber N (2002) Production, properties and specificity of a new bacterial L-fucose and D-arabinose-binding lectin of the plant aggressive pathogen Ralstonia solanacearum, and its comparison to related plant and microbial lectins. J Biochem 132(2): 353-358.
13. Wu AM, Wu JH, Singh T, Singha B, Sudakevitz D, et al. (2009) Multivalent human blood group $\mathrm{ABH}$ and Lewis glycotopes are key recognition factors for a LFuc $>$ Man binding lectin from phytopathogenic Ralstonia solanacearum. Biochim Biophys Acta 1790(4): 249-259.

14. Kostlánová N, Mitchell EP, Lortat-Jacob H, Oscarson S, Lahmann M, et al. (2005) The fucose-binding lectin from Ralstonia solanacearum: A new type of beta-propeller architecture formed by oligomerization and interacting with fucoside, fucosyllactose, and plant xyloglucan. J Biol Chem 280(30): 27839-27849.

15. Cozens D, Read RC (2012) Anti-adhesion methods as novel therapeutics for bacterial infection. Expert Rev Anti Infect Ther. 10(12): 1457-1468.

16. Bucior I, Abbott J, Song Y, Matthay MA, Engel JN, et al. (2013) Sugar administration is an effective adjunctive therapy in the treatment of Pseudomonas aeruginosa pneumonia. Am J Physiol Lung Cell Mol Physiol 305(5): L352-L363.

17. Newburg DS (2009) Neonatal protection by an innate immune system of human milk consisting of oligosaccharides and glycans. J Anim Sci 87(13 Suppl): 26-34.

18. Gerland B, Goudot A, Pourceau G, Meyer A, Dugas V, et al. (2012) Synthesis of a Library of Fucosylated Glycoclusters and Determination of their Binding toward Pseudomonas aeruginosa Lectin B (PAIIL) Using a DNA-Based Carbohydrate Microarray. Bioconjug Chem 23(8):1534-1547.

19. Reymond JL, Bergmann M, Darbre T (2013) Glycopeptide dendrimers as Pseudomonas aeruginosa biofilm inhibitors. Chem Soc Rev 42(11): 4814-4816.

20. Doknic D, Abramo M, Sutkeviciute L, Reinhardt A, Guzzi C, et al. (2013) Synthesis and characterization of linker-armed fucose-based glycomimetics. Eur J Org Chem 24: 5303-5314.

21. Deguise I, Lagnoux D, Roy R (2007) Synthesis of glycodendrimers containing both fucoside and galactoside residues and their binding properties to PA-IL and PA-IIL lectins from Pseudomonas aeruginosa. New J Chem 31(7): 1321-1331.

22. Imberty A, Chabre YM, Roy R (2008) Glycomimetics and glycodendrimers as high affinity microbial anti- adhesins. Chemistry 14(25): 7490-7499.

23. Chabre YM, Giguère D, Blanchard B, Rodrigue J, Rocheleau $S$, et al. (2011) Combining glycomimetic and multivalent strategies toward designing potent bacterial lectin inhibitors. Chemistry 17(23): 65456562.

24. Lerrer B, Gilboa-Garber N (2001) Interaction of Pseudomonas aeruginosa galactophilic lectin PA-IL with pigeon egg white glycoproteins. FEMS Immun Med Microbiol 32(1): 33-36.

25.Lerrer B, Gilboa-Garber N (2001) Interactions of Pseudomonas aeruginosa PA-IIL lectin with quail egg white glycoproteins. Can J Microbiol 47(12): 1095-1100.

26. Lesman-Movshovich E, Lerrer B, Gilboa-Garber N (2003) Blocking of Pseudomonas aeruginosa lectins by human milk glycans. Can J Microbiol 49(3): 230-235.

27.Zinger-Yosovich KD, Sudakevitz D, Iluz D, Gilboa-Garber N (2011) Analyses of diverse mammals' milk and lactoferrin glycans using five pathogenic bacterial lectins. Food Chem 124(4): 1335-1342.

28. Gilboa-Garber N, Zinger-Yosovich KD, Lerrer B (2009) Comparison of the antibacterial adhesion activities of honey and royal jelly in blocking lectins of the soil-borne pathogens Pseudomonas aeruginosa, 
Chromobacterium violaceum and Ralstonia solanacearum - to those of milk model. J ApiProduct ApiMedical Sci 1(3): 82-89.

29. Zinger-Yosovich KD, Gilboa-Garber N (2009) Blocking of Pseudomonas aeruginosa and Ralstonia solanacearum lectins by plant and microbial branched polysaccharides used as food additives. J Agric Food Chem 57(15): 6908-6913.

30. Rachmaninov O, Zinger-Yosovich KD, Gilboa-Garber N (2012) Preventing Pseudomonas aeruginosa and Chromobacterium violaceum infections by anti-adhesion-active components of edible seeds. Nutr J 11(10).

31. Rachmaninov O, Zinger-Yosovich KD, Gilboa-Garber N (2012) Preventing Ralstonia solanacearum adhesion with glycans from cashew, cocoa, coffee, pumpkin, and tomato seed extract. Can J Microbiol 58(7): 856-862.

32. Lane JA, Mehra RK, Carrington SD, Hickey RM (2010) The food glycome: A source of protection against pathogen colonization in the gastrointestinal tract. Int J Food Microbiol 142(1-2): 1-13.

33. Gilboa-Garber N (1994) Pseudomonas aeruginosa PA-I and PA-II lectins. In: Beuth J \& Pulverer G (Eds.), Lectin Blocking: New Strategies for the Prevention and Therapy of Tumor Metastasis and Infectious Diseases. Gustav Fischer Verlag, New York, pp. 44-58.

34. Shoaf K, Mulvey GL, Armstrong GD, Hutkins RW (2006) Prebiotic galactooligosaccharides reduce adherence of enteropathogenic Escherichia coli to tissue culture cells. Infect Immun 74(12): 69206928.

35. Wilson IBH, Zeleny R, Kolarich D, Staudacher E, Stroop CJM, et al. (2001) Analysis of Asn-linked glycans from vegetable foodstuffs: widespread occurrence of Lewis a, core $\alpha 1$,3-linked fucose and xylose substitutions. Glycobiology 11(4): 261-274.

36. Altmann F (2007) The role of protein glycosylation in allergy. Int Arch Allergy Immunol 142(2): 99-115.

37. Clark AT, Anagnostou K, Ewan PW (2007) Cashew nut causes more severe reactions than peanut: case-matched comparison in 141 children. Allergy 62(8): 913-916.

38. Lee CY, Shallenberger RS, Vittum MT (1970) Free sugars in fruits and vegetables. Food Sci: Food Sci Technol 1: 1-12.

39. Chen CP, Song SC, Gilboa-Garber N, Chang KS, Wu AM (1998) Studies on the binding site of the galactose-specific agglutinin PA-IL from Pseudomonas aeruginosa. Glycobiology 8(1): 7-16.
40.Zeleny R, Altmann F, Praznik W (1999) Structural characterization of the N-linked oligosaccharides from tomato fruit. Phytochemistry 51(2): 199-210.

41. Kimura Y, Matsuo S, Tsurusaki S, Kimura M, Hara-Nishimura I, et al. (2002) Subcellular localization of endo-beta-N-acetylglucosaminidase and high-mannose type free N-glycans in plant cell. Biochim Biophys Acta 1570(1): 38-46.

42. Puhlmann J, Bucheli E, Swain MJ, Dunning N, Albersheim P, et al. (1994) Generation of monoclonal antibodies against plant cell-wall polysaccharides. 1. Characterization of a monoclonal antibody to a terminal alpha-(1-2)-linked fucosyl-containing epitope. Plant Physiol 104(2): 699-710.

43. Lesman-Movshovich E, Gilboa-Garber N (2003) Pseudomonas aeruginosa lectin PA-IIL as a powerful probe for human and bovine milk analysis. J Dairy Sci 86(7): 2276-2282.

44. PerretS, Sabin C, Dumon C, Pokorna M, Gautier C, etal. (2005) Structural basis for the interaction between human milk oligosaccharides and the bacterial lectin PA-IIL of Pseudomonas aeruginosa. Biochem J 389(Pt 2): 325-332.

45. Schroder R, Nicolas P, Vincent SJF, Fischer M, Reymond S, et al. (2001) Purification and characterisation of a galactoglucomannan from kiwifruit (Actinidia deliciosa). Carbohyd Res 331(3): 291-306.

46. Sutherland P, Hallett L, Redgwell R, Benhamou N, MacRae E (1999) Localization of cell wall polysaccharides during kiwifruit (Actinidia deliciosa) ripening. Int J Plant Sci 160(6): 1099-1109.

47. Deters AM, Schroder KR, Hensel A (2005) Kiwi fruit (Actinidia chinensis L.) polysaccharides exert stimulating effects on cell proliferation via enhanced growth factor receptors, energy production, and collagen synthesis of human keratinocytes, fibroblasts, and skin equivalents. J Cell Physiol 202(3): 717-722.

48. Fitchette-Laine AC, Gomord V, Cabanes M, Michalski JC, Saint Macary $M$, et al. (1997) N-glycans harboring the Lewis a epitope are expressed at the surface of plant cells. Plant J 12(6): 1411-1417.

49. Melo NS, Nimtz M, Conradt HS, Fevereiro PS, Costa J (1997) Identification of the human Lewis(a) carbohydrate motif in a secretory peroxidase from a plant cell suspension culture (Vaccinium myrtillus L.) FEBS Lett 415(2): 186-191. 